\title{
Discours
}

Revue de linguistique, psycholinguistique et

informatique. A journal of linguistics, psycholinguistics

and computational linguistics

$6 \mid 2010$

Le fonctionnement en discours des énoncés averbaux autonomes

\section{Anaphore associative et relations de cohérence : une expression particulière de la relation Assertion-Indice}

Associative anaphora and coherence relations: a particular expression of the relation Claim-Evidence

\section{Mathilde Salles}

\section{OpenEdition}

\section{Journals}

Édition électronique

URL : http://journals.openedition.org/discours/7739

DOI : $10.4000 /$ discours. 7739

ISSN : 1963-1723

Éditeur :

Laboratoire LATTICE, Presses universitaires de Caen

Référence électronique

Mathilde Salles, « Anaphore associative et relations de cohérence : une expression particulière de la relation Assertion-Indice », Discours [En ligne], 6 | 2010, mis en ligne le 28 septembre 2010, consulté le 19 avril 2019. URL : http://journals.openedition.org/discours/7739; DOI : 10.4000/discours.7739

Ce document a été généré automatiquement le 19 avril 2019.

\section{c) (†) $९$}

Discours est mis à disposition selon les termes de la licence Creative Commons Attribution - Pas d'Utilisation Commerciale - Pas de Modification 4.0 International. 


\section{Anaphore associative et relations de cohérence : une expression particulière de la relation Assertion- Indice}

Associative anaphora and coherence relations: a particular expression of the relation Claim-Evidence

Mathilde Salles

\section{Introduction}

1 On a souvent souligné l'influence réciproque entre relation de cohérence et choix référentiel, en présentant notamment l'emploi d'expressions "surspécifiées "1 , c'est-àdire plus spécifiées que nécessaire pour l'identification référentielle, à la fois comme un effet du relâchement de la cohérence interpropositionnelle et comme un moyen d'exprimer ce relâchement. Par exemple, Li et Thompson (1979) remarquent que, en chinois, l'occurrence d'un pronom à la place d'une forme zéro (forme non marquée) est liée au degré de connexion entre les propositions. On a d'autre part parfois remarqué l'affinité entre telle ou telle relation lexicale et telle ou telle relation de cohérence : entre l'antonymie et la relation de Contraste, l'hyponymie et les relations de Généralisation, d' Exemplification ou d'Élaboration (cf. Cornish, 2006 et 2009a et b), ou encore entre la relation partie-tout et la relation d'Élaboration (cf. Asher, Lascarides, 1996 ; Cornish, 2006 et 2009a et b).

2 L'anaphore associative constitue un mode d'intégration particulier de certaines relations lexicales dans le discours, relations méronymiques et locatives entre autres (cf. Kleiber, 2001). Cette manière de présenter les référents «associatifs » a-t-elle des effets sur les relations de cohérence, des effets qui lui seraient propres et qui ne seraient pas seulement dus à l'emploi de telle ou telle relation lexicale? 
On commencera, dans la section 1, par illustrer le rôle de certaines marques linguistiques pour l'interprétation des relations de cohérence, avant de s'attacher plus particulièrement à deux d'entre elles : une relation lexicale, la relation partie-tout, et une relation anaphorique, l'anaphore associative (section 2). La troisième section s'efforcera de montrer que la coïncidence observée à la section 2 entre l'anaphore associative et la relation Assertion-Indice (Cornish, 2009a et b) n'est nullement fortuite, mais qu'elle est due au mode référentiel propre à cette relation anaphorique. Une comparaison avec un mode concurrent, l'anaphore possessive, le soulignera. Pour conclure, la dernière section réaffirmera cette influence particulière de l'anaphore associative en l'élargissant aux séquences (Adam, 2004) descriptives : la spécificité référentielle de l'anaphore associative soutient non seulement la dimension argumentative de la relation Assertion-Indice, mais aussi, plus généralement, celle des descriptions, qui sont rarement réduites à de simples Élaborations d'entité (Prévot et al., 2009).

\section{Marques linguistiques des relations de cohérence}

Les connecteurs apparaissent comme un des moyens les plus efficaces d'identifier une relation de cohérence entre deux phrases (cf., par exemple, Knott, Dale, 1994 ; Knott, Sanders, 1998; ou encore les tableaux proposés par Kehler, $2002: 19$ et 21), même si, fait maintes fois signalé, la correspondance n'est pas biunivoque. Mais les connecteurs ne sont pas les seules marques linguistiques concourant à l'identification des relations de cohérence et l'influence d'autres marques, tels, par exemple, les types de procès, les temps verbaux ou les relations anaphoriques, est régulièrement soulignée. Cornish (2006, 2009a et b) en a dressé un inventaire, comprenant, outre les connecteurs, les relations lexicales entre les prédicats des phrases ou entre certains de leurs arguments, le type de procès exprimé (état, activité, accomplissement, achèvement) par ces prédicats, les temps verbaux et les relations anaphoriques. On pourrait aussi évoquer, à la suite de Taboada (2006 : 573-574), la ponctuation à l'écrit, l'intonation, voire les gestes, à l'oral. Ou encore certaines constructions attributives spécificationnelles utilisant un nom sous-spécifié (cf. Legallois, 2006) en position initiale (e.g. Le résultat / La conséquence est que...), ce type de nom étant susceptible de jouer un rôle analogue à celui des connecteurs à la fois dans ces constructions attributives (voir Legallois, 2006) et dans des emplois détachés (e. g. Résultat, rien ne va plus, ou encore, relevé par Taboada, $2006: 583$, "The result: Kidder will focus on rich individual investisors [...]»).

Prenons l'exemple de la relation d'Élaboration, que Kelher (2002: 18) illustre avec la séquence suivante :

[1] A young aspiring politician was arrested in Texas today. John Smith, 34, was nabbed in a Houston law firm while attempting to embezzle funds for his campaign.

Cette relation de cohérence suppose à la fois identité et nouveauté, une double face que le complément à la définition de Hobbs $(1990: 95)^{2}$ :

Inférer la même proposition $\mathrm{P}$ à partir de l'assertion de $\mathrm{S}^{0}$ comme de $\mathrm{S}^{1}$, proposé par Cornish (2009b : 169) :

De plus $\mathrm{S}^{1}$ doit permettre d'ajouter d'autres détails à la proposition commune inférable de chaque assertion, et $\mathrm{e}^{1} \subseteq \mathrm{e}^{0}$ ('événement principal évoqué par $\mathrm{S}^{1}$ est une partie de celui dénoté par $\mathrm{S}^{0}$ ), 
souligne parfaitement bien.

7 Conformément à la définition de Hobbs, les deux phrases de l'exemple [1] conduisent à la même proposition - «un jeune politicien a été arrêté » - et, conformément à la spécification de Cornish, la deuxième phrase ajoute détails et informations nouvelles à cette proposition, en l'occurrence ici des précisions concernant le principal protagoniste (son nom et son âge) et les circonstances de l'événement (le lieu et le motif de l'arrestation). La relation d'Élaboration qui unit ces deux phrases est supportée par un ensemble de marques linguistiques - relations lexicales, voix et temps verbaux exprimant l'identité (du côté des procès) ou l'inclusion (du côté des localisations spatiales) : la synonymie des deux verbes arrest et nab, tous deux au prétérit passif, et la relation d'inclusion spatiale ${ }^{3}$ qui unit les toponymes Texas / Houston.

Les indices linguistiques peuvent être moins nombreux, mais extrêmement déterminants pour l'interprétation d'autres types de marques (comme les types de procès) et pour l'interprétation de la séquence entière. Ainsi, dans l'exemple [2a], repris à Schnedecker (1997: 85 et $2003: 117$ ), l'utilisation du nom propre, à la place d'un pronom comme en [2b], permet d'exprimer une tout autre relation de cohérence entre les deux phrases, une relation de cohérence qui s'accompagne d'une interprétation différente des types de procès :

[2a] Betty a soif. Betty boit.

(extrait d'un résumé du film Betty de Chabrol)

[2b] Betty a soif. Elle boit

Comme le précise Schnedecker (2003 : 117), dans la version originale - avec nom propre «le rapport de causalité [qui s'établirait naturellement dans une version pronominale ${ }^{4}$ ] disparaît, et [...] corollairement, on infère de l'enchaînement que Betty est alcoolique ». En [2a], la deuxième situation n'est plus présentée comme la conséquence de la première (Betty a soif donc elle boit), mais comme une élaboration de celle-ci, les deux phrases autorisant la même inférence «Betty est alcoolique » et recevant toutes deux une lecture habituelle. Corrélativement à ces différences de relations de cohérence, on interprétera différemment les types de procès de ces deux enchaînements: dans la version pronominale, l'état exprimé par la première phrase («avoir soif») cause l'activité exprimée par la deuxième phrase ( «boire »), alors que, dans la version avec nom propre, l'état exprimé par la première phrase est redoublé par l'état exprimé par la deuxième phrase («boire» encore, mais avec cette fois une lecture habituelle). Les deux occurrences de boit s'opposent par la dynamicité : le procès, dynamique dans la version pronominale, ne l'est plus dans la version avec nom propre, et le sujet n'est plus Agent, mais Siège du procès. L'identité entre les types de procès (états) et les rôles sémantiques attribués aux noms propres sujets (Sièges) des deux phrases Betty a soif. Betty boit permet une interprétation élaborante de la séquence ${ }^{5}$, alors que le décalage entre types de procès (état / activité) et rôles sémantiques (Siège / Agent) dans la version pronominale favorise au contraire une interprétation consécutive.

\section{Relation lexicale et / ou relation anaphorique?}

L'anaphore associative (désormais AA) réunit deux types d'indices parmi ceux qu'évoque Cornish, relation lexicale (méronymie, localisation fonctionnelle, etc.) ${ }^{6}$ et relation anaphorique. La question est de savoir si ces indices sont vraiment complémentaires ou si 
un seul joue véritablement un rôle pour l'interprétation des relations de cohérence. Par exemple, les liens entre relation partie-tout (au sens large) et relation d'Élaboration soulignés à la fois par Asher, Lascarides (1996) et Cornish (2006 et 2009a et b), ne se manifestent pas nécessairement à travers des AA. À cet égard, la distinction de FabriciusHansen, Behrens (2001) entre e-élaboration - e[ventuality]-elaboration, portant sur l'événement évoqué par la phrase élaborée - et i-élaboration - i[ndividual]-elaboration portant, elle, sur les seuls participants, les actants de cet événement ${ }^{-7}$ pourrait être décisive. L'e-élaboration, spécification de la situation entière, s'appuierait plus volontiers sur des relations partie-tout entre les prédicats (éventuellement associés à certains de leurs arguments), l'un décrivant un sous-événement de l'autre, en maintenant une constante référentielle du côté de l'actant ou des actants principaux, comme c'est le cas dans l'exemple d'Asher, Lascarides (1996: 89-90) :

[3a] Guy enjoyed a lovely meal.

$[3 b]$ He ate salmon.

[3c] He devored lots of cheese.

11 L'Agent ne varie pas, mais les procès de [b] et [c] - ate salmon, devored lots of cheese ${ }^{8}$ précisent la première proposition en décrivant diverses parties du repas apprécié par Guy.

12 L'i-élaboration, quant à elle, aurait, en tant que spécification des actants, une préférence pour les relations partie-tout (au sens large encore une fois) ${ }^{9}$ nominales, susceptibles de s'exprimer sous la forme d'une AA, comme dans l'exemple proposé par Kleiber, Vassiliadou (2007: 155 et $2009: 189)$ :

[4] Nous entrâmes dans un village. L'église était située sur une butte.

Dans cet exemple, la deuxième phrase effectue une i-élaboration de l'entité "village ", introduite dans la première phrase, via un de ses composants, l'église ${ }^{10}$.

Mais le partage des différentes relations partie-tout entre i-élaboration et e-élaboration n'est pas si rigide et relations partie-tout nominales et AA peuvent, semble-t-il, servir à la fois une i-élaboration et une e-élaboration. Ainsi, dans l'exemple suivant :

[5] Hugues recommençait chaque soir le même itinéraire, suivant la ligne des quais, d'une démarche indécise, un peu voûté déjà, quoiqu'il eût seulement quarante ans. Mais le veuvage avait été pour lui un automne précoce. Les tempes étaient dégarnies, les cheveux pleins de cendre grise. (G. Rodenbach, Bruges-la-morte, Bruxelles, Babel, 1989 : 25)

les anaphoriques associatifs les tempes, les cheveux, et les prédicats qui leur sont associés, en même temps qu'ils spécifient telle ou telle propriété de l'individu - ce qui relève d'une i-élaboration -, s'interprètent comme autant de signes du vieillissement prématuré du personnage - ce qui relève cette fois d'une e-élaboration de la proposition précédente, une spécification de la situation exprimée dans cette proposition et, plus encore, on va le voir, d'une relation Claim-Evidence ou Assertion-Indice (Cornish, 2009a et b).

Dans les exemples [4] et [5], l'AA a-t-elle, en elle-même, en dehors des relations lexicales sur lesquelles elle s'appuie, une influence sur la détermination des relations de cohérence? Les relations de cohérence seraient-elles les mêmes avec, par exemple, une anaphore possessive (cf. [6] et [7]) ?

[6] Nous entrâmes dans un village. Son église était située sur une butte. 
[7] Hugues recommençait chaque soir le même itinéraire, suivant la ligne des quais, d'une démarche indécise, un peu voûté déjà, quoiqu'il eût seulement quarante ans. Mais le veuvage avait été pour lui un automne précoce. Ses tempes étaient dégarnies, ses cheveux pleins de cendre grise. observé en [5] lui est-il particulier?

\section{Anaphore associative et relation Assertion-Indice}

\subsection{La relation Assertion-Indice (Claim-Evidence)}

17 Cette relation, que Cornish (2009a et b) classe parmi les relations d'Expansion (dites aussi relations de Ressemblance), avec les relations d'Élaboration, Parallèle et Contraste, comprend une partie élaborante [cf. 1) ci-dessous], "sémantique", concernant les contenus propositionnels des unités reliées, et une partie argumentative, " pragmatique » ${ }^{11}$, concernant cette fois les relations entre les actes illocutoires associés à ces unités [cf. 2) ci-dessous] :

Assertion-Indice :

1) Inférer $P$ de l'assertion de $S^{0}$ ainsi que de $S^{1}$, où $S^{1}$ ajoute d'autres détails à $P$ et $\mathrm{e}^{1}$

$\subseteq \mathrm{e}^{0}\left[\mathrm{e}^{1}\right.$ et $\mathrm{e}^{0}$ sont les événements principaux évoqués par $\mathrm{S}^{1}$ et $\left.\mathrm{S}^{0}\right](=$ la relation

Élaboration)

2) Interpréter $S^{1}$ comme rendant plus convaincante l'hypothèse du locuteur qui correspond à l'assertion de $\mathrm{S}^{0}$.

(Cornish, 2009b : 169)

18 La première partie de la définition de Cornish correspond ainsi à la définition de la relation Élaboration, fondamentalement « sémantique » (cf. Cornish, 2009b : 172, note 19), c'est-à-dire se fondant en priorité sur le contenu sémantique des unités. En l'occurrence, il s'agit de spécifier le contenu sémantique d'une première unité en ajoutant un ou des détails supplémentaires sur la situation décrite (e-élaboration) ou/et sur l'un des participants (i-élaboration). La deuxième partie de sa définition souligne la valeur argumentative de la deuxième unité, destinée à accroître la conviction de l'interlocuteur ou du lecteur : « la seconde des deux unités impliquées, écrit Cornish (2009b : 175), devra être interprétable comme rendant l'assertion de la première plus convaincante pour l'allocutaire ou le lecteur ». Cette fonction argumentative de la seconde unité est analogue à celle de la relation Démonstration (Evidence) de Mann et Thompson (1988), relation de présentation, ayant pour but d'augmenter la croyance de l'interlocuteur ou du lecteur.

L'exemple choisi par Cornish (2009a et b) pour illustrer cette relation de cohérence est l'un des exemples les plus célèbres de la littérature consacrée aux relations de cohérence :

[8] John can open Bill's safe. He knows the combination.

(Hobbs, $1979: 78$ et $1990: 96$ )

Cette séquence, qui permet à Hobbs de souligner l'influence des relations de cohérence sur l'interprétation des anaphoriques, est, selon son auteur, un exemple d'Élaboration: "By assuming that 'he' refers to John and that the combination is the combination of Bill's safe, we have the same proposition $P$ and have thus established the elaboration relation (and solved some coreference problems as a by-product [...])». Chaque phrase 
nous conduit en effet à inférer que John est capable d'ouvrir le coffre-fort de Bill (ici la proposition inférable $\mathrm{P}$ correspond à l'assertion de $\mathrm{S}^{0}$; cf. Hobbs, 1990 : 96). Et $\mathrm{S}^{1}$ (la deuxième phrase) implique $\mathrm{S}^{0}$ (la première) : si John connaît la combinaison du coffre-fort de Bill, alors il peut ouvrir ce coffre-fort. Mais, à cause de la proximité entre cette inférence et une relation causale ( $\mathrm{S}^{1}$ cause $\mathrm{S}^{0}$ ), Hobbs admet qu'Élaboration et Explication peuvent se mêler ici : c'est aussi parce qu'il connaît la combinaison du coffre-fort de Bill que John est capable d'ouvrir ce coffre-fort.

21 L'interprétation de Cornish (2009a et b) est différente : outre sa fonction élaborante, la deuxième phrase peut fournir à l'interlocuteur ou au lecteur une preuve de l'assertion effectuée en $\mathrm{S}^{0}$, une raison d'y croire. Le fait que John connaisse la combinaison est un argument pour accréditer l'hypothèse sur ses capacités formulée dans la première phrase. $\mathrm{S}^{1}$ acquiert ici une dimension démonstrative, la relation de cohérence est pragmatique (et non strictement sémantique, comme semble l'être la relation d' Explication de Hobbs) ${ }^{12}$. L'insertion d'un connecteur comme après tout (after all en anglais) ou en effet entre les deux phrases permettrait, selon Cornish (2009b), de souligner cette interprétation (John peut ouvrir le coffre-fort de Bill. Après tout/En effet, il connaît la combinaison).

\subsection{Anaphores associatives comme Indices (Evidences)}

22 Outre l'exemple [5] déjà donné, dans lequel les anaphoriques associatifs et les propriétés prédiquées («Les tempes étaient dégarnies, les cheveux pleins de cendre grise ») s'interprétaient comme des preuves, des signes du vieillissement du personnage, autrement dit comme des Indices de l'Assertion effectuée dans la phrase précédente ( Mais le veuvage avait été pour lui un automne précoce ») ${ }^{13}$, d'autres exemples confirment cette affinité entre AA et relation Assertion-Indice. Ces exemples présentent des similitudes remarquables, qui concourent à l'identification d'une forme particulière d'Assertion-Indice, fondée sur une séquence descriptive plus ou moins élaborée :

[9] M. Lebigre tenait un fort bel établissement, d'un luxe tout moderne. Placé à l'encoignure droite de la rue Rambuteau, flanqué de quatre petits pins de Norvège dans des caisses peintes en vert, il faisait un digne pendant à la grande charcuterie des Quenu-Gradelle. Les glaces claires laissaient voir la salle, ornée de guirlandes de feuillages, de pampres et de grappes, sur un fond vert tendre. Le dallage était noir et blanc, à grands carreaux. Au fond, le trou béant de la cave s'ouvrait sous l'escalier tournant, à draperie rouge, qui menait au billard du premier étage. Mais le comptoir surtout, à droite, était très riche, avec son large reflet d'argent poli.

(Zola, Le ventre de Paris, Paris, Gallimard, 2002 : 168)

[10] Ce visage noir était anguleux et creusé dans tous les sens. Le menton était creux ; les tempes étaient creuses ; les yeux étaient perdus en de jaunâtres orbites. Les os maxillaires, rendus saillants par une maigreur indescriptible, dessinaient des cavités au milieu de chaque joue. Ces gibbosités, plus ou moins éclairées par les lumières, produisaient des ombres et des reflets curieux qui achevaient d'ôter à ce visage les caractères de la face humaine.

(Balzac, Sarrasine ; cité par J.-M. Marandin, 1986 : 85)

[11] [...] il se posséda mieux et demanda au domestique de lui apporter une glace ; elle lui glissa aussitôt des mains ; il se reconnaissait à peine ; la figure était couleur de terre, les lèvres boursouflées et sèches, la langue ridée, la 


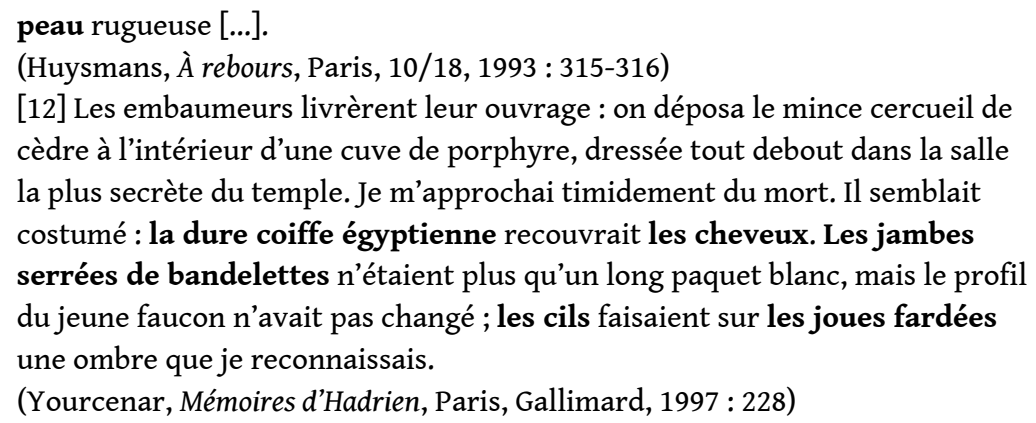

Comme l'exemple [5], tous ces exemples présentent une série de prédicats statifs, qui, en même temps qu'ils «e-élaborent » un prédicat statif initial (« faisait un digne pendant à la grande charcuterie des Quenu-Gradelle » en [9] ; «était anguleux et creusé dans tous les sens » en [10] ; «se reconnaissait à peine » en [11] ; « semblait costumé » puis "n'avait pas changé » en [12]), s'interprètent comme autant de preuves particulières (au sens étymologique) d'une assertion valant plus généralement pour le référent. Ainsi le luxe de l'établissement de $\mathrm{M}$. Lebigre, en cela digne pendant de la charcuterie des Quenu-Gradelle, est confirmé par les propriétés de ses différentes parties ${ }^{14}$. De même, la métamorphose physique du référent en [11], son travestissement, en même temps qu'une forme d'immutabilité en [12], sont confirmés par les descriptions d'états de certaines parties du corps. Enfin, la somme des prédications particulières de [10] («Le menton était creux; les tempes étaient creuses; les yeux étaient perdus en de jaunâtres orbites. Les os maxillaires, rendus saillants par une maigreur indescriptible, dessinaient des cavités au milieu de chaque joue. »), toutes de creux et de bosses, non seulement précise (i. e. élabore) l'état décrit dans la première phrase, mais encore le prouve par l'exemple ${ }^{15}$. Les AA de ces exemples soutiennent un type d'argument inductif, puisqu'il s'agit, à chaque fois, de certifier l'état d'une entité globale par l'état de telle(s) ou telle(s) de ses parties. Et, dans chacun de ces exemples, on pourrait insérer le connecteur en effet (proposé par Cornish, 2009b, comme indice possible de la relation Assertion-Indice) avant la séquence anaphorique associative; e.g. [13] et [14] pour les exemples [10] et [12] respectivement :

[13] Ce visage noir était anguleux et creusé dans tous les sens. En effet, le menton était creux; les tempes étaient creuses; les yeux étaient perdus en de jaunâtres orbites. Les os maxillaires, rendus saillants par une maigreur indescriptible, dessinaient des cavités au milieu de chaque joue. Ces gibbosités, plus ou moins éclairées par les lumières, produisaient des ombres et des reflets curieux qui achevaient d'ôter à ce visage les caractères de la face humaine.

[14] Les embaumeurs livrèrent leur ouvrage : on déposa le mince cercueil de cèdre à l'intérieur d'une cuve de porphyre, dressée tout debout dans la salle la plus secrète du temple. Je m'approchai timidement du mort. Il semblait costumé. En effet $^{16}$, la dure coiffe égyptienne recouvrait les cheveux. Les jambes serrées de bandelettes n'étaient plus qu'un long paquet blanc, mais le profil du jeune faucon n'avait pas changé ; en effet $^{17}$, les cils faisaient sur les joues fardées une ombre que je reconnaissais.

\subsection{Anaphore associative et anaphore possessive}

La relation Assertion-Indice, lorsqu'elle repose sur une évocation, voire une énumération de parties, trouve-t-elle une meilleure expression dans l'AA ou est-elle indifférente au 
mode de présentation des parties? On se contentera ici de comparer les effets produits par l'alternance, lorsque celle-ci est possible ${ }^{18}$, entre l'AA et l'anaphore possessive.

L'absence de toute mention du tout (sous la forme d'un déterminant possessif ou d'un complément du nom), qui caractérise l'AA, se traduit par une forme d'aliénation de la partie. En effet, avec un défini associatif (le $N$ ) à la place, par exemple, d'un possessif (son $N$ ) ou d'une description définie complète (le $N d u N$ ), la partie devient, selon Azoulay (1978: 29), « l'élément important du discours », elle est « considéré[e] dans son existence propre ». Plus précisément :

Par opposition à l'individu d'une description complète correspondante [Le $N \mathrm{du} N$ ], celui d'une description $L e N$ en anaphore associative apparaît comme déjà délimité, isolé ou détaché. Il est en quelque sorte aliéné. (Kleiber, 1999 : 85 et 2001 : 242)

À partir de cette propriété du défini associatif, Kleiber (1999 et 2001) définit une contrainte, qu'il nomme " condition d'aliénation », sur le type de référent autorisant l'AA :

Condition d'aliénation: le référent d'une anaphore associative doit être présenté ou donné comme aliéné par rapport au référent de l'antécédent. (Kleiber 1999: 85 et $2001: 242)$

Cette condition, combinée à un principe dit "de congruence ontologique», permet d'expliquer certaines restrictions de l'AA avec des noms de matière, de forme et, dans une moindre mesure, des noms de parties du corps. Les remarques d'Azoulay (1978) citées plus haut avaient d'ailleurs pour but d'expliquer l'inacceptabilité de l'AA avec des noms de parties du corps (inacceptabilité cependant infirmée par plusieurs de nos exemples); cf. [15], par opposition à [16] :

[15] *Jacques est tombé du premier étage. Les pieds sont cassés.

(Azoulay, $1978: 27$ )

[16] J'ai fait tomber la petite table. Les pieds sont cassés.

(Azoulay, $1978: 26$ )

Selon Azoulay (1978 : 29) :

Il est difficile de parler d'un objet dont le rapport à un être humain est étroit sans mentionner cet être humain. L'énoncé [15] impliquerait [à cause de l'emploi du seul défini] que le pied est l'élément important du discours, qu'il est considéré dans son existence propre et que Jacques n'est pas affecté par ce qu'on en dit, ce qui n'est pas plausible. D'où l'inacceptabilité de [15]. On pourrait avancer un principe qui stipulerait qu'un « thème » humain doit toujours être présent dans la phrase.

Des exemples tels que [5], [10], [11] et [12] montrent toutefois que la présence d'un «thème » humain dans la phrase n'est pas toujours nécessaire et, corollairement, qu'une partie du corps peut être l'élément important du discours, qu'elle peut être considérée dans son existence propre, c'est-à-dire qu'une partie ontologiquement inaliénable peut autoriser une aliénation discursive. Cette possibilité dépend, selon Kleiber et al. (1994: 24), de la « capacité du prédicat à aliéner, ou autrement dit à autonomiser une partie d'un tout ». Ainsi, dans l'exemple suivant :

[17] Jean a été étranglé. Le cou est en effet tout couvert de bleus.

(Kleiber et al., $1994: 24$ )

le verbe étrangler possède cette capacité aliénante vis-à-vis de la partie " cou »; étrangler, écrivent Kleiber et al. (1994 : 24), « a pour effet d'isoler et de rendre saillante la partie cou d'un individu $»^{19}$. Mais c'est aussi la capacité des prédicats suivants à présenter la ou les parties comme des preuves de ce qui a été préalablement asserté qui justifie cette 
aliénation, cet effet de focalisation, de gros plan (l'image est utilisée par Kleiber, 1999 et 2001), cette promotion de la partie au statut d'élément important du discours. Car l'exemple forgé par Kleiber et al. (1994), ce n'est sûrement pas une coïncidence, illustre encore une relation Assertion-Indice, dont l'interprétation est même renforcée par la présence du connecteur en effet. Et, si l'on compare les différents exemples proposés sous [18] et [19], cette relation de cohérence paraît déterminante pour l'AA :

[18a] Jean a été étranglé. Les yeux sont révulsés.

[18b] Jean a été étranglé. ? Les yeux sont fermés.

[18c] Jean a été étranglé. Ses yeux sont fermés.

[19a] Ce visage noir était anguleux et creusé dans tous les sens. Les yeux étaient perdus en de jaunâtres orbites. (à partir de Balzac, Sarrasine; cf. exemple [10])

[19b] Ce visage noir était anguleux et creusé dans tous les sens. ? Les yeux étaient vifs ${ }^{20}$. n'accèdera plus aussi facilement au statut de preuve, d'argument. Autrement dit, l'anaphore possessive ne constituera pas un moyen aussi efficace que l'AA d'introduire des Indices dans le cadre d'une relation Assertion-Indice (elle nécessitera davantage la présence d'un marqueur comme en effet) et sera préférée pour exprimer d'autres relations. Par exemple, une anaphore possessive à la place de l'AA de l'exemple [5] :

[20] Mais le veuvage avait été pour lui un automne précoce. Ses tempes étaient dégarnies, ses cheveux pleins de cendre grise.

ne permet plus aux parties du corps d'indiquer seules, i. e. sans la présence d'un marqueur supplémentaire tel le connecteur en effet, une relation Assertion-Indice. En l'absence d'un tel marqueur, la séquence s'interprétera plutôt en termes de Cause-Conséquence (ou encore relation Résultat), c'est-à-dire comme une relation de cohérence permettant l'insertion de connecteurs comme $d u$ coup, donc, ainsi, de ce fait entre les deux phrases: Mais le veuvage avait été pour lui un automne précoce. Du coup / De ce fait, ses tempes étaient dégarnies, ses cheveux pleins de cendre grise. La valeur explicative se trouve en quelque sorte inversée par rapport à la version originale : en [20], le fait que le veuvage ait été un automne précoce pour le personnage explique la présence de certaines marques physiques; en [5], les signes du vieillissement du personnage - promus éléments importants du discours grâce à l'AA ${ }^{21}$ - justifient l'Assertion initiale, selon laquelle le veuvage avait été pour lui un automne précoce.

\section{L'importance de l'anaphore associative pour les descriptions}

L'AA, par son effet focalisant, permet de souligner la dimension argumentative, démonstrative des descriptions, qui ne sont pas de purs ornements. Cf. à ce sujet les précieux rappels d'Adam (1993: 45) sur ces séquences mal aimées: «la rhétorique 
antique avait déjà assigné au descriptif des limites très précises : la description était subordonnée aux besoins de la démonstration ou de la narration ». Et les descriptions qui seraient de pures i-élaborations ou Élaborations d'entité se révèlent beaucoup plus rares que ne le suggèrent Prévot et al. (2009). Même les appositions "ordinaires $»^{22}$ décrivant un état sont souvent chargées d'un contenu plus riche que celui d'une simple spécification d'entité. Le plus souvent, il s'agit d'une valeur explicative [21] ou concessive [22] :

[21] Ma mère, institutrice, veut le secondaire pour sa petite-fille.

(Duras, L'Amant ; exemple repris à Gardes Tamine, 2004 : 182)

[22] On a récompensé $M^{\text {lle }}$ Ausserac, une petite rouée.

(Colette, « Au concert »; Gardes Tamine, 2004 : 182)

Avec l'AA, le processus argumentatif, on l'a vu, est de type inductif, allant du particulier (les parties-preuves) au général (le tout, Assertion générale prouvée par le raisonnement inductif, puisqu'une somme de propriétés particulières est censée démontrer une propriété du tout).

L'AA constituera donc un moyen privilégié d'énumérer des parties. Les descriptions de lieux (le café de M. Lebigre en [9], redonné en [23]) et d'objets (la pièce montée de [24]) en sont particulièrement friandes :

[23] M. Lebigre tenait un fort bel établissement, d'un luxe tout moderne. Placé à l'encoignure droite de la rue Rambuteau, flanqué de quatre petits pins de Norvège dans des caisses peintes en vert, il faisait un digne pendant à la grande charcuterie des Quenu-Gradelle. Les glaces claires laissaient voir la salle, ornée de guirlandes de feuillages, de pampres et de grappes, sur un fond vert tendre. Le dallage était noir et blanc, à grands carreaux. Au fond, le trou béant de la cave s'ouvrait sous l'escalier tournant, à draperie rouge, qui menait au billard du premier étage. Mais le comptoir surtout, à droite, était très riche, avec son large reflet d'argent poli.

(Zola, Le ventre de Paris, Paris, Gallimard, 2002 : 168)

[24] On avait été chercher un pâtissier à Yvetot, pour les tourtes et les nougats. Comme il débutait dans le pays, il avait soigné les choses; et il apporta, lui-même, au dessert, une pièce montée qui fit pousser des cris. À la base, d'abord, c'était un carré de carton bleu figurant un temple avec portiques, colonnades et statuettes de stuc tout autour, dans des niches constellées d'étoiles en papier doré; puis se tenait au second étage un donjon en gâteau de Savoie, entouré de menues fortifications en angélique, amandes, raisins secs, quartiers d'oranges; et enfin, sur la plate-forme supérieure, qui était une prairie verte où il y avait des rochers avec des lacs de confitures et des bateaux en écales de noisettes, on voyait un petit Amour, se balançant à une escarpolette de chocolat, dont les deux poteaux étaient terminés par deux boutons de rose naturels, en guise de boules, au sommet. (Flaubert, Madame Bovary, Paris, Gallimard, 1985 : 53 ; cité par Adam, 1993 : 99 et $2005: 118$ )

Comme le souligne Adam (1993 et 2005), les descriptions se caractérisent par une présence importante d'organisateurs textuels - organisateurs spatiaux (à gauche / à droite, devant / derrière, dessus/dessous, en haut/en bas, au nord / au sud...), temporels et / ou énumératifs (d'abord, ensuite, (et) puis, (et) enfin, alors, après...). Dans les exemples [23] et [24], ces organisateurs spatiaux sont des anaphoriques associatifs ou des locutions adverbiales formellement très proches ${ }^{23}$ : avec des AA locatives ou méronymiques (sous l'escalier tournant, au second étage, sur la plate-forme supérieure), on trouve en effet des locutions adverbiales comme au fond, en [23], à la base, au sommet, en [24], toutes 
construites avec un «nom de localisation interne » (Borillo, 1988 et 1999, Aurnague, 1989 et 1996). L'anaphore possessive, elle, ne semble guère mise à contribution pour ces structurations spatiales, ce qui peut s'expliquer à la fois par son incapacité à promouvoir la partie (en l'occurrence organisateur spatial) au premier plan et par sa tendance à "décentrer le point d'attache", tendance soulignée par Kleiber et al. (1994: 56): l'anaphore possessive, contrairement à l'anaphore définie associative, ne peut en effet "que pivoter autour de l'axe référentiel dont elle dépend structurellement, tant et si bien qu'elle opère à très courte portée, sinon sa manifestation dans des concaténations du type son $N 1$... son $N 2 \ldots$ son $N 3 . .$. risque de décentrer le point d'attache ».

\section{Conclusion}

L'AA permet de décrire une entité d'une manière singulière, en détachant ses parties, ce qui, on l'a constaté, peut avoir des incidences sur la dimension argumentative des relations de cohérence. Cette influence sur la dimension argumentative des relations de cohérence explique la relative facilité (compte tenu de la nature des entités désignées, $a$ priori peu disposées à l'aliénation) avec laquelle les noms de parties du corps entrent en AA : dans nombre d'exemples, l'aliénation de parties inaliénables, leur promotion au statut d'éléments importants du discours se justifie par la capacité des prédicats à présenter ces parties comme des preuves d'une assertion initiale, autrement dit se justifie dans le cadre d'une relation Assertion-Indice. Elle explique aussi, plus généralement, le rôle de cette anaphore pour la description, rôle à peine esquissé ici et qui mériterait une étude, voire des études plus approfondies, car les effets de l'AA sur les séquences descriptives sont multiples : si l'AA est parfois «spectaculaire ", au service de véritables hypotyposes ${ }^{24}$, sa fonction peut aussi être celle d'un simple organisateur textuel, lorsqu'elle apparait dans des constructions prépositionnelles comme celles des exemples [23] et [24].

\section{BIBLIOGRAPHIE}

ADAM, J.-M. 1993. La description. Que sais-je ? Paris : PUF.

ADAM, J.-M. 2004. La linguistique textuelle. Des genres de discours aux textes. Paris : Nathan Université. ADAM, J.-M. 2005. La linguistique textuelle. Introduction à l'analyse textuelle des discours. Paris : Armand Colin.

ADLER, S. 2008. Événementialité et partitivité dans les séquences [PREP LE NOM DE] temporelles. Langages $169: 67-81$.

ASHER, N., LASCARIDES, A. 1996. Lexical disambiguation in a discourse context. In J. PUSTEJOVSKY., B. BOGURAEV (eds), Lexical Semantics : the problem of polysemy. Oxford : Clarendon Press : 69-108.

AURnAGUE, M. 1989. Catégorisation des objets dans le langage : les noms de localisation interne. Cahiers de grammaire $14: 1-21$. 
AURNAGUE, M. 1996. Les noms de localisation interne : tentative de caractérisation sémantique à partir de données du basque et du français. Cahiers de lexicologie 69 (2) : 159-192.

AZOUlay, A. 1978. Article défini et relations anaphoriques en français. Recherches linguistiques 7 : $5-45$.

BORILlo, A. 1988. Le lexique de l'espace : les noms et les adjectifs de localisation interne. Cahiers de grammaire $13: 1-22$.

BORILLO, A. 1999. Partition et localisation spatiale : les noms de localisation interne. Langages 136 : 53-75.

CORNISH, F. 2006. Relations de cohérence en discours : critères de reconnaissance, caractérisation et articulation cohésion-cohérence. Corela : Organisation des textes et cohérence des discours. D. LEGALLOIS (ed.). Accessible en ligne sur http://edel.univ-poitiers.fr/corela/document.php?id=1280

CORNISH, F. 2009a. Inter-sentential anaphora and coherence relations in discourse : a perfect match. Language Sciences $31: 572-592$.

CORNISH, F. 2009b. Le rôle des anaphores dans la mise en place des relations de cohérence dans le discours : l'hypothèse de J.R. Hobbs. Journal of French Language Studies : Relations de cohérence et fonctionnement des anaphores 19 (2). F. CORNISH (ed.) : 159-181.

FABRICIUS-HANSEN, C., BEHRENS, B. 2001. Elaboration and related discourse relations viewed from an interlingual perspective. Sprik reports $13: 1-34$. Accessible en ligne sur http://www.hf.uio.no/ german/sprik.

FONTANIER, P. 1977. Les figures du discours (1821-1830). Paris : Flammarion.

GARDES TAMINE, J. 2004. Pour une grammaire de l'écrit. Paris : Belin

HALLIDAY, M.A.K., HASAN, R. 1976. Cohesion in English. London : Longman.

HOBBS, J.R. 1979. Coherence and coreference. Cognitive Science 3 : 67-90.

HOBBS, J.R. 1990. Literature and Cognition. Stanford University : CLSI Publications. Chapitre 5 : The Coherence and Structure of Discourse : 83-114.

KEHLER, A. 2002. Coherence, Reference, and the Theory of Grammar. Stanford University : CSLI Publications.

KLEIBER, G. 1994. Anaphores et pronoms. Louvain-la-Neuve : Duculot.

KLEIBER, G. 1999. Anaphore associative et relation partie-tout : condition d'aliénation et principe de congruence ontologique. Langue française 122 : 70-100.

KLEIBER, G. 2001. L'anaphore associative. Paris : PUF.

KLEIBER, G, SCHNEDECKER, C., UJMA, L. 1994. L'anaphore associative, d'une conception l'autre. In C. SCHNEDECKER, M. CHAROLLES, G. KLEIBER, J. DAVID (eds), L'anaphore associative. Recherches linguistiques, XIX. Paris : Klincksieck : 5-64.

KLEIBER, G., VASSILIADOU, H. 2007. Sur les approches intuitives de la relation d'Élaboration. Scolia22 : 147-161.

KLEIBER, G., VASSILIADOU, H. 2009. Sur la relation d'Élaboration : des approches intuitives aux approches formelles. Journal of French Language Studies : Relations de cohérence et fonctionnement des anaphores 19 (2). F. CORNISH (ed.) : 183-205. 
KNOTT, A., DALE, R. 1994. Using linguistic phenomena to motivate a set of coherence relations. Discourse Processes 18 (1) : 35-62.

KNOTT, A., SANDERS, T. 1998. The classification of coherence relations and their linguistic markers : An exploration of two languages. Journal of pragmatics $30: 135-175$.

LEGALLOIS, D. 2006. Quand le texte signale sa structure : la fonction textuelle des noms sousspécifiés. Corela : Organisation des textes et cohérence des discours. D. LEGALLOIS (ed.). Accessible en ligne sur http://edel.univ-poitiers.fr/corela/document.php?id=1288.

LI, C.N., THOMPSON, S.A. 1979. Third-Person Pronouns and Zero-Anaphora in Chinese Discourse. Syntax and Semantics 12. J.P. KIMBALl (ed.). New York : Academic Press : 311-335.

MANN, W.C., THOMPSON, S.A. 1988. Rhetorical Structure Theory : Toward a functional theory of text organization. Text 8 (3) : 243-281.

MARANDIN, J.-M. 1986. Ce est un autre. L'interprétation anaphorique du syntagme démonstratif. Langages $81: 75-109$.

MARANDIN, J.-M. 1998. Grammaire de l'incidence. Accessible en ligne sur http://www.llf.cnrs.fr/Gens/ Marandin/GrammaireDeL_incidence.htm.

MARTIN, J.R. 1983. Conjonction: The logic of English text. In J.S. PETÖFI, E. SÖZER (eds), Micro and macroconnexity of texts. Hamburg : Buske : 1-72.

MILNER, J.-C. 1978. De la syntaxe à l'interprétation. Paris : Seuil.

PRÉVOT, L., VIEU, L., ASHER, N. 2009. Une formalisation plus précise pour une annotation moins confuse : la relation d'Élaboration d'entité. Journal of French Language Studies : Relations de cohérence et fonctionnement des anaphores 19 (2). F. CORNISH (ed.) : 207-228.

SANDERS, T., SPOOREN, W., NOORDMAN, L. 1992a. Toward a taxonomy of coherence. Discourse Processes $15: 1-35$.

SANDERS, T., SPOOREN, W., NOORDMAN, L. 1992b. Coherence relations in a cognitive theory of discourse representation. Cognitive linguistics 4 (2) : 93-133.

SCHNEDECKER, C. 1997. Nom propre et chaînes de référence. Paris : Klincksieck.

SCHNEDECKER, C. 2003. La question du nom propre répété dans la théorie dite du centrage et ses problèmes. French Language Studies 13 : 105-134.

TABOADA, M. 2006. Discourse markers as signals (or not) of rhetorical relations. Journal of

Pragmatics $38: 567-592$.

VAN DIJK, T. A. 1979. Pragmatic connectives. Journal of Pragmatics $3: 447-456$.

VONK, W., HUSTINX, L., SIMONS, W. 1992. The use of referential expressions in structuring discourse.

Language and Cognitive Processes 7 (3-4) : 301-333.

WinStON, M.E., CHAFfin, R., HERMAnN, D. 1987. A Taxonomy of Part-Whole Relations. Cognitive Science $11: 417-444$.

\section{NOTES}

1. « Overspecified », chez Vonk et al. (1992). 
2. Définition qui, elle, est davantage tournée vers le même. Pour une revue des différentes définitions de la relation d'Élaboration, on se reportera à Kleiber, Vassiliadou (2007) et (2009).

3. Traitée comme un type de relation méronymique - la relation lieu-surface (place-area) - par Winston et al. (1987).

4. Et cela à cause des propriétés sémantiques et référentielles du pronom de troisième personne qui, en saisissant le référent en continuité avec ce qui l'a rendu saillant dans le contexte précédent (cf. Kleiber, 1994), aura tendance à convoquer la connexion la plus forte entre les phrases.

5. La caractérisation de la relation d'Élaboration par Fabricius-Hansen, Behrens (2001:10-11) met bien en exergue ces conditions de ressemblance : « e1 and e2 [e=eventuality] must belong to the same situation type (accomplishment / achievement, activity [...]) and have compatible argument structures, their Agent referents must be identical, or more generally: referents having corresponding roles with respect to e1 and e2 must be identical, the temporal and spatial location of e2 must be the same as or a subpart of the location of e1, etc. ». Sur l'homogénéité entre types de procès (ou éventualités), voir aussi Prévot et al. (2009).

6. Je n'évoquerai ici que deux des catégories de la typologie des AA établie par Kleiber (2001) : les AA méronymiques et les AA locatives.

7. Ou encore, la distinction entre Élaboration et Élaboration d'entité, chez Prévot et al. (2009).

8. Les syntagmes verbaux entiers (verbe et complément) ici; les arguments des verbes lovely meal / salmon, lots of cheese jouant, comme le souligne un relecteur, un rôle crucial pour la relation partie-tout et l'e-élaboration.

9. Au sens large incluant, outre les relations méronymiques au sens strict, les relations de localisation fonctionnelle de Kleiber (2001), comme celle qui unit église à village en [4].

10. Cette i-élaboration pourrait évidemment aussi se faire plus directement, sans relation partietout, en attribuant une propriété au village: e.g. Il était charmant, avec une anaphore coréférentielle à la place de l'anaphore associative de [4].

11. Au sens de Sanders et al. (1992a et b). L'opposition entre source sémantique et source pragmatique de la cohérence constitue l'un des quatre primitifs fondant leur classification des relations de cohérence. Comme ils le signalent eux-mêmes (1992a: 8), cette distinction est comparable à celle de van Dijk (1979) - déjà en termes de "sémantique » vs " pragmatique » - ou encore à celle qu'utilisent Halliday et Hasan (1976) ou Martin (1983) entre relations externes ou internes à la situation de communication. Cf. aussi, chez Mann et Thompson (1988), la distinction entre relations de contenu et relations de présentation.

12. Une relation purement sémantique ne produirait probablement pas la suite la plus cohérente après une assertion utilisant l'auxiliaire modal can, qui, ainsi que le précise Cornish (2009a et b), contribue à conférer une valeur d'« Assertion » à la première unité.

13. On pourrait ici aussi proposer d'insérer le connecteur en effet entre les deux phrases.

14. Au sens large toujours, puisqu'on trouve à la fois des AA méronymiques (e.g. le premier étage) et des AA locatives (e.g. le comptoir).

15. Cette description méronymique a une double influence argumentative, puisqu'elle conduit en outre à l'Assertion finale (dans laquelle le SN ces gibbosités, étudié par Marandin, 1986 : 86, clôt la séquence descriptive en réunissant les différentes parties « fragmentées » par l'anaphore définie) sur le caractère monstrueux, la dimension fantastique de ce visage.

16. Les deux points du texte original («Il semblait costumé: la dure coiffe égyptienne [...]») semblent d'ailleurs jouer un rôle comparable à celui du connecteur en effet ici.

17. La répétition de en effet à si peu d'intervalle n'est évidemment pas du meilleur « effet ».

18. Comme le note Kleiber (2001: 309), le possessif est toujours possible lorsqu'on a affaire à des relations méronymiques (e.g. une voiture/son moteur, un arbre/ses racines), mais pas toujours lorsqu'il s'agit de relations locatives (e.g. un village / son église, une cuisine / ? son réfrigérateur; ces deux exemples sont repris par Kleiber, $2001: 309$, note 1). 
19. Pour une présentation et une explication plus développée du fonctionnement des noms de parties du corps en AA, cf. Kleiber (1999 et 2001).

20. Il paraît plus difficile de proposer, en face de [19b], une séquence possessive - ses yeux étaient vifs - comme on l'a fait en [18c]. La propension du possessif à sélectionner un "possesseur» animé, tout particulièrement avec un nom de parties du corps, rend en effet peu probable une interprétation de ses yeux comme « les yeux du visage noir ».

21. Alors que dans la version possessive, l'individu reste au premier plan, celui-ci passe en quelque sorte au second plan dans la version anaphorique associative (il n'est d'ailleurs pas mentionné dans l'énoncé), à cause précisément des gros plans opérés sur certaines parties de son corps.

22. Prévot et al. (2009 : 218), à la suite de Marandin (1998), opposent les appositions ordinaires, incidentes à un $\mathrm{SN}$, et les appositions qualitatives, incidentes à la phrase (Marandin reconnaît aussi des appositions ordinaires incidentes à la phrase tel le SN initial de l'exemple suivant: Événement exceptionnel, le dictateur a donné un sauf-conduit aux cheminots grévistes). Entre autres propriétés syntaxiques (voir Marandin, 1998, qui s'appuie ici sur les travaux de Milner, 1978), les appositions ordinaires ne peuvent être éloignées de leur SN d'incidence. Ce test de déplacement, que retiennent Prévot et al. (2009), permet de confirmer que les appositions des exemples [21] et [22] sont bien des appositions ordinaires.

23. On peut d'ailleurs hésiter à les identifier comme des locutions adverbiales plutôt que comme des syntagmes prépositionnels incluant un SN défini anaphorique. La même question se pose pour les expressions temporelles à la fin, au début, qu'étudie Adler (2008).

24. L'effet de gros plan des définis associatifs favorise l'hypotypose, qui « peint les choses d'une manière si vive et énergique, qu'elle les met en quelque sorte sous les yeux, et fait d'un récit ou d'une description, une image, un tableau ou même une scène vivante » (Fontanier, 1977 : 390).

\section{RÉSUMÉS}

Les relations anaphoriques et les relations lexicales comptent parmi les marques linguistiques des relations de cohérence. L'anaphore associative, qui constitue un mode d'intégration particulier de certaines relations lexicales dans le discours, réunit ces deux types de marques.La question est de savoir si celles-ci sont complémentaires et quel rôle elles jouent dans l'interprétation des relations de cohérence. On verra en particulier que cette manière de présenter les référents " associatifs" a des effets sur la dimension argumentative de certaines relations de cohérence, et qu'elle explique le lien privilégié qu'on peut observer entre l'anaphore associative et la relation Assertion-Indice (Cornish, 2009a et b). Une comparaison avec un mode référentiel concurrent, l'anaphore possessive, soulignera que ces effets sont propres au mode référentiel de l'anaphore associative, et qu'ils ne sont pas simplement liés à l'emploi de telle ou telle relation lexicale.

Anaphoric relations and lexical relations can guide the hearer or reader in the recognition of coherence relations. These two kinds of markers occur with associative anaphora. Are they complementary? What part do they take in the interpretation of coherence relations? In this paper, I suggest that this anaphoric way to introduce some lexical relations (in particular partwhole relations) affects the argumentative nature of coherence relations and explains the affinity we can observe between associative anaphora and the relation Claim-Evidence (Cornish, 
2009a and b). A comparison with possessive anaphora, a competitive way of introducing partwhole relations, emphasizes that this effect is peculiar to associative anaphora and not due to the use of the lexical relation itself.

INDEX

Mots-clés : anaphore associative, anaphore possessive, Assertion-Indice, Élaboration, relation partie-tout, relations de cohérence

Keywords : associative anaphora, Claim-Evidence, coherence relations, Elaboration, part-whole relation, possessive anaphora

\section{AUTEUR}

\section{MATHILDE SALLES}

CRISCO, EA 4255, Université de Caen

UFR des Sciences de l'Homme, Université de Caen, Esplanade de la Paix, 14032 Caen cedex mathilde.salles@unicaen.fr 